\title{
The Equivalence Postulate of Quantum Mechanics, Dark Energy, and the Intrinsic Curvature of Elementary Particles
}

\author{
Alon E. Faraggi \\ Department of Mathematical Sciences, University of Liverpool, Liverpool L69 7ZL, UK \\ Correspondence should be addressed to Alon E. Faraggi; alon.faraggi@liv.ac.uk
}

Received 9 April 2013; Accepted 23 April 2013

Academic Editor: Shi-Hai Dong

Copyright ( 2013 Alon E. Faraggi. This is an open access article distributed under the Creative Commons Attribution License, which permits unrestricted use, distribution, and reproduction in any medium, provided the original work is properly cited.

\begin{abstract}
The equivalence postulate of quantum mechanics offers an axiomatic approach to quantum field theories and quantum gravity. The equivalence hypothesis can be viewed as adaptation of the classical Hamilton-Jacobi formalism to quantum mechanics. The construction reveals two key identities that underlie the formalism in Euclidean or Minkowski spaces. The first is a cocycle condition, which is invariant under D-dimensional Möbius transformations with Euclidean or Minkowski metrics. The second is a quadratic identity which is a representation of the $D$-dimensional quantum Hamilton-Jacobi equation. In this approach, the solutions of the associated Schrödinger equation are used to solve the nonlinear quantum Hamilton-Jacobi equation. A basic property of the construction is that the two solutions of the corresponding Schrödinger equation must be retained. The quantum potential, which arises in the formalism, can be interpreted as a curvature term. The author proposes that the quantum potential, which is always nontrivial and is an intrinsic energy term characterising a particle, can be interpreted as dark energy. Numerical estimates of its magnitude show that it is extremely suppressed. In the multiparticle case the quantum potential, as well as the mass, is cumulative.
\end{abstract}

\section{Introduction}

Understanding the synthesis of quantum mechanics and gravity is an important challenge in theoretical physics. The main effort in this endeavour is in the framework of string theory. The primary advantage of string theory is that it gives rise to the gauge and matter ingredients of elementary particle physics and predicts the number of degrees of freedom needed to obtain a consistent theory. String theory therefore enables the construction of quasirealistic models and the development of a phenomenological approach to quantum gravity. The state of the art in this regard is the heterotic string models in the free fermionic formulation, which reproduce the matter content of the minimal supersymmetric standard model and preserve its unification picture [1-5]. Despite its phenomenological success string theory does not provide a framework for a rigorous formulation of quantum gravity from fundamental principles.

Important characteristics of string theories are its various perturbative and nonperturbative dualities. Indeed, one of the interesting approaches to formulating string theory aims to promote $T$-duality [6] to a manifest property of the formalism [7]. T-duality can be viewed heuristically as phasespace duality in compact space.

A formalism that aims to promote phase-space duality to a level of a fundamental principle was followed in the context of the equivalence postulate approach to quantum mechanics [8-15]. The equivalence postulate of quantum mechanics hypothesises that all physical systems are equivalent under coordinate transformations. In particular, there should always exist a coordinate transformation connecting a physical system with a nontrivial potential $V$ and energy $E$, to the one with $V-E=0$. Conversely, any allowed physical state should arise by a coordinate transformation from the state with $V-E=0$. Thus, nontrivial states arise from the inhomogeneous term stemming from the transformation of the trivial state under coordinate transformations. It is then seen that both the definability of the phase-space duality, and consistency of the equivalence postulate for all physical states require the modification of classical mechanics by quantum mechanics. More precisely, the phase-space duality, as well as the equivalence postulate, is ill defined in classical mechanics for the trivial state, for which Hamilton's generating function $S_{0}$ is a constant or a linear function of the coordinate. The 
quantum modification removes this state from the space of allowed solutions and enables the consistency of the equivalence postulate, as well as definability of phase-space duality, for all physical states.

In this paper, I argue that another feature of the equivalence postulate approach is the existence of dark energy, which arises as an intrinsic property of elementary particles. This property of elementary particles arises from the quantum potential, which is never vanishing and corresponds to an intrinsic curvature associated with elementary particles.

\section{Equivalence Postulate of Quantum Mechanics}

In this section I review the equivalence postulate approach to quantum mechanics. It is important to emphasise that the equivalence postulate formulation of quantum mechanics does not entail an interpretation or a modification, but rather a mathematically rigorous derivation of quantum mechanics from a fundamental postulate. In this respect the equivalence postulate provides an axiomatic approach to quantum mechanics and quantum field theories. The formalism has some reminiscences of Bohm's approach to quantum mechanics in the sense that both approaches may be regarded as a modification of the classical Hamilton-Jacobi formalism. However, aside from this superficial similarity the two approaches are fundamentally distinct.

A view of the equivalence postulate approach is obtained by an analogy with the classical Hamilton-Jacobi formalism. The classical Hamilton equations of motion are given by

$$
\dot{p}=-\frac{\partial H}{\partial q}, \quad \dot{q}=\frac{\partial H}{\partial p},
$$

where $p$ and $q$ are the phase space variables and $H$ is the Hamiltonian. In classical mechanics the solution to the mechanical problem is obtained by performing canonical transformations to a new set of phase space variables

$$
q \longrightarrow Q(q, p, t), \quad p \longrightarrow P(q, p, t)
$$

such that the new Hamiltonian and Hamilton equations of motion are trivial $H(q, p) \rightarrow K(Q, P) \equiv 0$. Consequently, the transformed phase space coordinates are constants of the motion. The solution to this problem in classical mechanics is given by the classical Hamilton-Jacobi equation (CHJE) which for systems that conserve the total energy gives the classical stationary Hamilton-Jacobi equation (CSHJE)

$$
\frac{1}{2 m}\left(\frac{\partial S}{\partial q}\right)^{2}+V(q)=E,
$$

where $S(q)$ is a generating function. For the purpose of this paper it is sufficient to focus here on the stationary case. In performing these trivialising transformations the phase space variables are taken as independent variables. The functional dependence between them is only extracted after solving the CSHJE equation (3) from the relation

$$
p=\frac{\partial S(q)}{\partial q}
$$

Since the transformations (2) are invertible we also have the inverse transformation

$$
Q \longrightarrow q(Q, P, t), \quad P \longrightarrow p(Q, P, t) .
$$

However, the intrinsic property of quantum mechanics is that the phase space variables are not independent; that is, they satisfy the commutation relation:

$$
[\widehat{q}, \widehat{p}]=i \hbar \text {. }
$$

We therefore relax the condition that the phase space variable are independent in the application of the trivialising transformation; and, hence, that the transformations are canonical. We further assume the functional relation between the phasespace variables via the generating function (4). We can ask the following question: is it possible in classical mechanics to start with a system with a nontrivial Hamiltonian, that is with a given nonvanishing $W(q)$, and connect via some coordinate transformations to any other system. This demand dictates that the Hamilton-Jacobi equation retains its form under the coordinate transformations. From the form of (3) we see that this is not possible in classical mechanics. The reason is that in classical mechanics the trivial state, with $V(q)-E \equiv 0$, is a fixed state under the transformations. Insisting that all physical states, including the trivial one, are connected by coordinate transformations necessitates the modification of the CSHJE. The modification is given by the quantum stationary Hamilton-Jacobi equation (QSHJE)

$$
\frac{1}{2 m}\left(\frac{\partial S(q)}{\partial q}\right)^{2}+W(q)+Q(q)=0,
$$

where $W(q)=V(q)-E$. From the form of (7) it is seen that the combination $W(q)+Q(q)$ transforms as a quadratic differential under coordinate transformations, whereas each of the functions $W(q)$ and $Q(q)$ transform, as a quadratic differential up to an additive term; that is, under $q \rightarrow \widetilde{q}(q)$ we have:

$$
\begin{gathered}
W(q) \longrightarrow \widetilde{W}(\widetilde{q})=\left(\frac{\partial q}{\partial \widetilde{q}}\right)^{2} W(q)+(q ; \tilde{q}), \\
Q(q) \longrightarrow \widetilde{Q}(\widetilde{q})=\left(\frac{\partial q}{\partial \widetilde{q}}\right)^{2} Q(q)-(q ; \tilde{q}), \\
W(q)+Q(q)) \\
\longrightarrow(\widetilde{W}(\widetilde{q})+\widetilde{Q}(\widetilde{q}))=\left(\frac{\partial q}{\partial \widetilde{q}}\right)^{2}(W(q)+Q(q)) .
\end{gathered}
$$

All physical states with a nontrivial $W(q)$ then arise from the inhomogeneous part in the transformation of the trivial state $W^{0}\left(q^{0}\right) \equiv 0$; that is, $W(q)=\left(q^{0} ; q\right)$.

Considering the transformation $q^{a} \rightarrow q^{b} \rightarrow q^{c}$ versus $q^{a} \rightarrow q^{c}$ gives rise to the cocycle condition on the inhomogeneous term

$$
\left(q^{a} ; q^{c}\right)=\left(\frac{\partial q^{b}}{\partial q^{c}}\right)^{2}\left[\left(q^{a} ; q^{b}\right)-\left(q^{c} ; q^{b}\right)\right]
$$


The cocycle condition (9) underlies the equivalence postulate and embodies its underlying symmetries. In particular, it is invariant under the Möbius transformations

$$
\left(\gamma\left(q^{a}\right) ; q^{b}\right)=\left(q^{a} ; q^{b}\right),
$$

where

$$
\gamma(q)=\frac{A q+B}{C q+D}
$$

and $\left(\begin{array}{ll}A & B \\ C & D\end{array}\right) \in \mathrm{GL}(2, C)$. In one dimension the cocycle condition (9) uniquely defines the Schwarzian derivative up to a constant and a coboundary term. Specifically, one obtains $\left(q^{a} ; q^{b}\right)=-\beta\left\{q^{a}, q^{b}\right\} / 4 m$, where $\{f, q\}=f^{\prime \prime \prime} / f^{\prime}-$ $3\left(f^{\prime \prime} / f^{\prime}\right)^{2} / 2$ denotes the Schwarzian derivative and $\beta$ is a constant with the dimension of an action. We further have that $\{\gamma(q), q\} \equiv 0$.

The one-dimensional stationary case is instructive to reveal the symmetry properties that underlie quantum mechanics in the equivalence postulate formalism. In one dimension the unique solution of the problem is given in terms of the Schwarzian identity

$$
\left(\frac{\partial S(q)}{\partial q}\right)^{2}=\frac{\beta^{2}}{2}\left(\left\{\mathrm{e}^{(2 i / \beta) S}, q\right\}-\{S, q\}\right)
$$

which embodies the equivalence postulate and leads to the Schrödinger equation. Making the identification

$$
\begin{gathered}
W(q)=V(q)-E=-\frac{\beta^{2}}{4 m}\left\{\mathrm{e}^{\left(i 2 S_{0}\right) / \beta}, q\right\}, \\
Q(q)=\frac{\beta^{2}}{4 m}\left\{S_{0}, q\right\},
\end{gathered}
$$

we have that $S_{0}$ is the solution of the quantum stationary Hamilton-Jacobi equation (QSHJE)

$$
\frac{1}{2 m}\left(\frac{\partial S_{0}}{\partial q}\right)^{2}+V(q)-E+\frac{\hbar^{2}}{4 m}\left\{S_{0}, q\right\}=0,
$$

From (13) and the properties of the Schwarzian derivative, we deduce that $S_{0}$, the solution of the QSHJE equation (15), is given by (see also [16-21]):

$$
\mathrm{e}^{(2 i / \beta) S_{0}}=\gamma(w)=\frac{A w+B}{C w+D}=\mathrm{e}^{i \alpha} \frac{w+i \bar{\ell}}{w-i \ell},
$$

where $\ell=\ell_{1}+i \ell_{2} ;\left\{\alpha, \ell_{1}, \ell_{2}\right\} \in R$. Here $w=\psi^{D} / \psi$ and $\psi^{D}$ and $\psi$ are two linearly independent solutions of a second order differential equation given by

$$
\left(-\frac{\beta^{2}}{2 m} \frac{\partial^{2}}{\partial q^{2}}+V(q)-E\right) \psi(q)=0,
$$

That is, $\psi^{D}$ and $\psi$ are the two solutions of the Schrödinger equation and we can identify $\beta \equiv \hbar$. We can note the relation between the Shrödinger equation in an alternative way. Inserting the solution

$$
\psi=\operatorname{Re}^{(i / \hbar) S_{0}}
$$

into the Schrödinger equation produces the two equations

$$
\begin{gathered}
\left(\partial_{q} S_{0}\right)^{2}+V(q)-E-\frac{\hbar^{2}\left(\partial_{q}^{2} R\right)}{(2 m R)}=0 \\
\partial_{q}\left(R^{2} \partial_{q} S_{0}\right)=0 .
\end{gathered}
$$

The continuity equation, (20), gives $R=1 / \sqrt{S_{0}^{\prime}}$ and consequently

$$
Q(q)=-\frac{\hbar^{2}}{2 m} \frac{\partial_{q}^{2} R}{R}=\frac{\hbar^{2}}{4 m}\left\{S_{0}, q\right\}
$$

and (19) corresponds to (15).

The steps taken in deriving the quantum Hamilton-Jacobi equation (15) from the Schrödinger equation are reminiscent of its derivation in the framework of Bohmian quantum mechanics. However, there is a crucial difference. While in Bohmian mechanics one identifies the solution (18) with the wave function, and hence $R^{2}$ with the probability density, it is noted that the equivalence postulate necessitates that both solutions $\psi$ and $\psi^{D}$ are kept in the formalism. This can be seen from the properties of the Schwarzian derivative that show that the trivialising transformation is given by

$$
q \longrightarrow q^{0} \equiv \gamma\left(\frac{\psi^{D}}{\psi}\right)
$$

that is, up to a Möbius transformation, $q^{0}$ is given by the ratio of the two solutions of the corresponding Schrödinger equation. Hence, in general the wave function in the equivalence postulate approach is given by

$$
\Psi(q)=R(q)\left(A \mathrm{e}^{(i / \hbar) S_{0}}+B \mathrm{e}^{-(i / \hbar) S_{0}}\right) .
$$

Furthermore, the equivalence postulate necessitates that $S_{0}(q) \neq A q+B$; that is, $S_{0}(q)$ cannot be a linear function of $q$. Strictly speaking the condition that the Schwarzian derivative is well defined only necessitates the weaker condition $S_{0}(q) \neq$ constant. However, the condition that the quantum potential is always nonvanishing leads to the stronger constraint $S_{0}(q) \neq A q+B$, which also follows from arguments concerning phase-space duality [8-14]. In the timedependent case the equivalence postulate implies that the wave function should always take the form

$$
\Psi(q, t)=R(q, t)\left(A \mathrm{e}^{(i / \hbar) S(q, t)}+B \mathrm{e}^{-(i / \hbar) S(q, t)}\right),
$$

where $S=S_{0}-E t$ in the stationary case. As discussed previously, consistency of the equivalence postulate of quantum mechanics dictates the necessity of employing the two solutions of the Schrödinger equation. This condition is well known in relativistic quantum mechanics and signals the departure from the single particle interpretation in nonrelativistic quantum mechanics to the multiparticle representation of quantum field theories. However, in nonrelativistic Bohmian mechanics, and in particular in the case of bound states, only the solution with the positive exponent is kept, 
which implies that in those cases in Bohmian mechanics $S_{0}=$ constant. The equivalence postulate approach, on the other hand, necessitates that both solutions are kept in the formalism and that $S_{0} \neq 0$ always. Consistency of the equivalence postulate further implies that the trivialising map, $q \rightarrow$ $\tilde{q}=\psi^{D} / \psi$, is continuous on the extended real line [8-14]. It is then seen that this condition is synonymous with the requirement that the physical solution of the corresponding Schrödinger equation admits a square integrable solution and selects the correct physical eigenstates for the bound states. In the relativistic case the inclusion of the "negative energy" states reveals the existence of antiparticles. The implications of incorporating the two solutions in the nonrelativistic case require more detailed scrutiny. It is noted that this decomposition of the wave function has been employed successfully in studies of molecular dynamics, $[22,23]$ and is referred to there as the bipolar decomposition.

The equivalence postulate formalism extends to the higher dimensional case both with respect to the Euclidean and Minkowski metrics [15]. The key to these extensions is the generalisations of the cocycle condition equation (9) and of the quadratic identity equation (12). Denote the transformations between two sets of coordinate systems by

$$
q \longrightarrow q^{v}=v(q)
$$

and the conjugate momenta by the generating function $S_{0}(q)$,

$$
p_{k}=\frac{\partial S_{0}}{\partial q_{k}}
$$

Under the transformations (25) we have $S_{0}^{v}\left(q^{v}\right)=S_{0}(q)$; hence

$$
p_{k} \longrightarrow p_{k}^{v}=\sum_{i=1}^{D} J_{k i} p_{i}
$$

where $J$ is the Jacobian matrix

$$
J_{k i}=\frac{\partial q_{i}}{\partial q_{j}^{v}} .
$$

Introducing the notation

$$
\left(p^{v} \mid p\right)=\frac{\sum_{k}\left(p_{k}^{v}\right)^{2}}{\sum_{k} p_{k}^{2}}=\frac{p^{t} J^{t} J p}{p^{t} p},
$$

the cocycle condition takes the form

$$
\left(q^{a} ; q^{c}\right)=\left(p^{c} \mid p^{b}\right)\left[\left(q^{a} ; q^{b}\right)-\left(q^{c} ; q^{b}\right)\right],
$$

which captures the symmetries that underlie quantum mechanics. It is shown that the cocycle condition (30) is invariant under $D$-dimensional Möbius transformations, which include dilatations, rotations, translations, and reflections in the unit sphere [15]. The quadratic identity, (12), is generalised by the basic identity

$$
\alpha^{2}\left(\nabla S_{0}\right)^{2}=\frac{\Delta\left(R \mathrm{e}^{\alpha S_{0}}\right)}{\operatorname{Re}^{\alpha S_{0}}}-\frac{\Delta R}{R}-\frac{\alpha}{R^{2}} \nabla \cdot\left(R^{2} \nabla S_{0}\right),
$$

which holds for any constant $\alpha$ and any functions $R$ and $S_{0}$. Then, if $R$ satisfies the continuity equation

$$
\nabla \cdot\left(R^{2} \nabla S_{0}\right)=0,
$$

and setting $\alpha=i / \hbar$, we have

$$
\frac{1}{2 m}\left(\nabla S_{0}\right)^{2}=-\frac{\hbar^{2}}{2 m} \frac{\Delta\left(R \mathrm{e}^{(i / \hbar) S_{0}}\right)}{R \mathrm{e}^{(i / \hbar) S_{0}}}+\frac{\hbar^{2}}{2 m} \frac{\Delta R}{R} .
$$

In complete analogy with the one-dimensional case we make identifications,

$$
\begin{gathered}
W(q)=V(q)-E=\frac{\hbar^{2}}{2 m} \frac{\Delta\left(R \mathrm{e}^{(i / \hbar) S_{0}}\right)}{R \mathrm{e}^{(i / \hbar) S_{0}}}, \\
Q(q)=-\frac{\hbar^{2}}{2 m} \frac{\Delta R}{R} .
\end{gathered}
$$

Equation (34) implies the $D$-dimensional Schrödinger equation

$$
\left[-\frac{\hbar^{2}}{2 m} \Delta+V(q)\right] \Psi=E \Psi
$$

and the general solution

$$
\Psi=R(q)\left(A \mathrm{e}^{(i / \hbar) S_{0}}+B \mathrm{e}^{-(i / \hbar) S_{0}}\right),
$$

is mandated by consistency of the equivalence postulate.

The equivalence postulate formalism generalises to the relativistic case as well. In this case, setting $q \equiv\left(c t, q_{1}, \ldots\right.$, $\left.q_{D-1}\right)$, with $q^{v}=v(q)$ a general transformation of the coordinates, we have

$$
\left(p^{v} \mid p\right)=\frac{\eta^{\mu \rho} p_{\mu}^{v} p_{\rho}^{v}}{\eta^{\mu \rho} p_{\mu} p_{\nu}}=\frac{p^{t} J \eta J^{t} p}{p^{t} \eta p},
$$

and $J$ is the Jacobian matrix

$$
J_{\rho}^{\mu}=\frac{\partial q^{\mu}}{\partial q^{v \rho}} .
$$

Furthermore, we obtain the cocycle condition

$$
\left(q^{a} ; q^{c}\right)=\left(p^{c} \mid p^{b}\right)\left[\left(q^{a} ; q^{b}\right)-\left(q^{c} ; q^{b}\right)\right],
$$

is invariant under $D$-dimensional Möbius transformations with respect to Minkowski metric. The quadratic identity in this case takes the form,

$$
\alpha^{2}(\partial S)^{2}=\frac{\square\left(R \mathrm{e}^{\alpha S}\right)}{R \mathrm{e}^{\alpha S}}-\frac{\square R}{R}-\frac{\alpha}{R^{2}} \partial \cdot\left(R^{2} \partial S\right),
$$

which holds for any constant $\alpha$ and any functions $R$ and $S$. Then, if $R$ satisfies the continuity equation $\partial\left(R^{2} \cdot \partial S\right)=0$, and setting $\alpha=i / \hbar$, we have

$$
(\partial S)^{2}=-\hbar^{2} \frac{\square\left(R \mathrm{e}^{(i / \hbar) S}\right)}{R \mathrm{e}^{(i / \hbar) S}}+\hbar^{2} \frac{\square R}{R} .
$$


Setting

$$
\begin{gathered}
W(q)=m c^{2}=-\hbar^{2} \frac{\square\left(\operatorname{Re}^{(i / \hbar) S}\right)}{R \mathrm{e}^{(i / \hbar) S}}, \\
Q(q)=\hbar^{2} \frac{\square R}{R}
\end{gathered}
$$

reproduces the relativistic Klein-Gordon equation

$$
\left(\hbar^{2} \square+m c^{2}\right) \Psi(q)=0
$$

with the general solution

$$
\Psi=R(q)\left(A \mathrm{e}^{(i / \hbar) S}+B \mathrm{e}^{-(i / \hbar) S}\right) .
$$

The equivalence postulate formalism also incorporates gauge interactions via the generalisation of the quadratic identities equations (12), (31), and (41), and of the cocycle conditions equations (9), (30), and (40). In the nonrelativistic stationary case the quadratic identity takes the form

$$
\begin{aligned}
\alpha^{2}\left(\nabla S_{0}+e A\right)^{2}= & \frac{(\nabla+e \alpha A)^{2}\left(R \mathrm{e}^{\alpha S_{0}}\right)}{\operatorname{Re}^{\alpha S_{0}}} \\
& -\frac{\Delta R}{R}-\frac{\alpha}{R^{2}} \nabla \cdot\left(R^{2}\left(\nabla S_{0}+e A\right)\right),
\end{aligned}
$$

and the cocycle condition equation (30) retains its form with

$$
\left(p^{v} p\right)=\frac{\left(p^{v}+e A^{v}\right)^{t}\left(p^{v}+e A^{v}\right)}{(p+e A)^{t}(p+e A)}
$$

whereas in the relativistic case they take the form

$$
\alpha^{2}(\partial S+e A)^{2}=\frac{D^{2} R e^{\alpha S}}{R e^{\alpha S}}-\frac{R}{R}-\frac{\alpha}{R^{2}} \partial \cdot\left(R^{2}(\partial S+e A)\right),
$$

where

$$
D_{\mu}=\partial_{\mu}+\alpha e A_{\mu}
$$

and the cocycle identity (30) retains its form with

$$
\left(p^{b} \mid p\right)=\frac{\left(p^{b}+e A^{b}\right)^{2}}{(p+e A)^{2}}=\frac{(p+e A)^{t} J \eta J^{t}(p+e A)}{(p+e A)^{t} \eta(p+e A)},
$$

and $J$ is the Jacobian matrix

$$
J_{v}^{\mu}=\frac{\partial q^{\mu}}{\partial q^{b^{\nu}}}
$$

We note the symmetry structure that underlies the formalism. Seeking further generalisation of this approach simply entails that this robust symmetry structure is retained. While the formalism has some reminiscences of Bohmian quantum mechanics, it is clearly distinct from it as no physical interpretation has so far been assigned to the wave function. Furthermore, consistency of the equivalence postulate formalism requires that the physical solutions are square integrable and selects the same eigenstates that in conventional quantum mechanics arise due to the probability interpretation of the wave function. In the framework of the equivalence postulate, the Schrödinger equation, and its solutions, is merely a device to find solutions of the nonlinear quantum Hamilton-Jacobi equation. To investigate the physical picture of the quantum potential in the equivalence postulate formalism we have to turn to its interpretation as a curvature term.

\section{The Quantum Potential as a Curvature Term}

The quantum stationary Hamilton-Jacobi equation (QSHJE), (7) can be viewed as a deformation of the classical stationary Hamilton-Jacobi equation (CSHJE), (3), by a "conformal factor." Noting that

$$
\left\{S_{0}, q\right\}=-\left(\partial_{q} S_{0}\right)^{2}\left\{q, S_{0}\right\}
$$

we have that the QSHJE (7) is equivalent to

$$
\frac{1}{2 m}\left(\frac{\partial S_{0}}{\partial q}\right)^{2}\left[1-\hbar^{2} U\left(S_{0}\right)\right]+V(q)-E=0,
$$

where $U\left(S_{0}\right)$ is the canonical potential

$$
U\left(S_{0}\right)=\frac{1}{2}\left\{q, S_{0}\right\},
$$

that arises in the framework of the $p-q$ duality [8-14]. Equation (53) can be written in the form

$$
\frac{1}{2 m}\left(\frac{\partial S_{0}}{\partial \widehat{q}}\right)^{2}+V(q)-E=0
$$

where

$$
\left(\frac{\partial q}{\partial \widehat{q}}\right)^{2}=\left[1-\hbar^{2} U\left(S_{0}\right)\right]
$$

or equivalently

$$
d \widehat{q}=\frac{d q}{\sqrt{1-\beta^{2}(q)}}
$$

with $\beta^{2}(q)=\hbar^{2} U\left(S_{0}\right)=\hbar^{2}\left\{q, S_{0}\right\} / 2$. Integrating (56) yields

$$
\widehat{q}=\int^{q} \frac{d x}{\sqrt{1-\beta^{2}(x)}} .
$$

It follows that

$$
\lim _{\hbar \rightarrow 0} \widehat{q}=q
$$

In the case of the trivial state $W^{0}\left(q^{0}\right) \equiv 0,(53)$ becomes

$$
\frac{1}{2 m}\left(\frac{\partial S_{0}^{0}}{\partial q^{0}}\right)^{2}\left[1-\hbar^{2} U\left(S_{0}\right)\right]=0
$$


whose solution is

$$
U\left(\frac{\hbar}{2 i} \ln \gamma\left(q^{0}\right)\right)=\frac{1}{\hbar^{2}},
$$

where

$$
\gamma\left(q^{0}\right)=\frac{A q^{0}+B}{C q^{0}+D},
$$

with $A D-B C \neq 0$. Equation (53) shows that the quantum potential can be regarded as a deformation of the space geometry. From (46) and (48) we note that in this respect the quantum potential is distinct from the interactions that arise from the gauge potentials, that can be viewed as a shift of the momentum. The quantum potential has a universal character, which is independent of gauge charges. Furthermore, Flanders showed that the Schwarzian derivative can be interpreted as a curvature of an equivalence problem for curves in $\mathbf{P}^{1}$ [24]. For that purpose, introduce a frame for $\mathbf{P}^{1}$, that is a pair $\mathbf{x}, \mathbf{y}$ of points in affine space $\mathbf{A}^{2}$ such that $[\mathbf{x}, \mathbf{y}]=1$, where

$$
[\mathbf{x}, \mathbf{y}]=\mathbf{x}^{t}\left(\begin{array}{cc}
0 & 1 \\
-1 & 0
\end{array}\right) \mathbf{y}=x_{1} y_{2}-x_{2} y_{1}
$$

is the area function, which has the $S L(2, \mathbf{R})$-symmetry

$$
[\widetilde{\mathbf{x}}, \widetilde{\mathbf{y}}]=[\mathbf{x}, \mathbf{y}] \text {, }
$$

where $\widetilde{\mathbf{x}}=R \mathbf{x}$ and $\widetilde{\mathbf{y}}=R \mathbf{y}$ with $R \in S L(2, \mathbf{R})$. Considering the moving frame $s \rightarrow\{\mathbf{x}(s), \mathbf{y}(s)\}$ and differentiating $[\mathbf{x}, \mathbf{y}]=1$ yield the structure equations

$$
\mathbf{x}^{\prime}=a \mathbf{x}+b \mathbf{y}, \quad \mathbf{y}^{\prime}=c \mathbf{x}-a \mathbf{y}
$$

where $a, b$, and $c$ depend on $s$. Given a map $\phi=\phi(s)$ from a domain to $\mathbf{P}^{1}$, one can choose a moving frame $\mathbf{x}(s), \mathbf{y}(s)$ in such way that $\phi(s)$ is represented by $\mathbf{x}(s)$. This map can be seen as a curve in $\mathbf{P}^{1}$. Two mappings $\phi$ and $\psi$ are said to be equivalent if $\psi=\pi \circ \phi$, with $\pi$ a projective transformation on $\mathbf{P}^{1}$.

Flanders considered two extreme situations. The first case corresponds to $b(s)=0$, for all $s$. In this case $\phi$ is constant. Taking the derivative of $\lambda \mathbf{x}$, for some $\lambda(s) \neq 0$, we have by (64) that $(\lambda \mathbf{x})^{\prime}=\left(\lambda^{\prime}+a \lambda\right) \mathbf{x}$. Choosing $\lambda \propto \exp \left[-\int_{s_{0}}^{s} d t a(t)\right] \neq 0$, we have $(\lambda \mathbf{x})^{\prime}=0$, so that $\lambda \mathbf{x}$ is a constant representative of $\phi$.

The other case is for $b$ never vanishing. There are only two inequivalent situations. The first one is when $b$ is either complex or positive. It turns out that it is always possible to choose the following "natural moving frame" for $\phi[24]$ :

$$
\mathbf{x}^{\prime}=\mathbf{y}, \quad \mathbf{y}^{\prime}=-k \mathbf{x} .
$$

In the other case, corresponding to $b$ real and negative, the natural moving frame for $\phi$ is

$$
\mathbf{x}^{\prime}=-\mathbf{y}, \quad \mathbf{y}^{\prime}=k \mathbf{x} .
$$

A characteristic property of the natural moving frame is that it is determined up to a sign with $k$ an invariant. Thus, for example, suppose that for a given $\phi$ there is, besides (66), the natural moving frame $\mathbf{x}_{1}^{\prime}=\mathbf{y}_{1}, \mathbf{y}_{1}^{\prime}=-k_{1} \mathbf{x}_{1}$. Since both $\mathbf{x}$ and $\mathbf{x}_{1}$ are representatives of $\phi$, we have $\mathbf{x}=\lambda \mathbf{x}_{1}$, so that $\mathbf{y}=$ $\mathbf{x}^{\prime}=\lambda^{\prime} \mathbf{x}_{1}+\lambda \mathbf{y}_{1}$ and $1=[\mathbf{x}, \mathbf{y}]=\lambda^{2}$. Therefore, $\mathbf{x}_{1}= \pm \mathbf{x}, \mathbf{y}_{1}=$ $\pm \mathbf{y}$, and $k_{1}=k[24]$.

Let us now review the derivation of Flanders formula for $k$. Consider $s \rightarrow \mathbf{z}(s)$ to be an affine representative of $\phi$ and let $\mathbf{x}(s), \mathbf{y}(s)$ be a natural frame. Then $\mathbf{z}=\lambda \mathbf{x}$ where $\lambda(s)$ is never vanishing. Now note that, since $\mathbf{z}^{\prime}=\lambda^{\prime} \mathbf{x}+\lambda \mathbf{y}$, we have that $\lambda$ can be written in terms of the area function $\left[\mathbf{z}, \mathbf{z}^{\prime}\right]=\lambda^{2}$. Computing the relevant area functions, one can check that $k$ has the following expression

$$
2 k=\frac{\left[\mathbf{z}, \mathbf{z}^{\prime \prime \prime}\right]+3\left[\mathbf{z}^{\prime}, \mathbf{z}^{\prime \prime}\right]}{\left[\mathbf{z}, \mathbf{z}^{\prime}\right]}-\frac{3}{2}\left(\frac{\left[\mathbf{z}, \mathbf{z}^{\prime \prime}\right]}{\left[\mathbf{z}, \mathbf{z}^{\prime}\right]}\right)^{2} .
$$

Given a function $z(s)$, this can be seen as the nonhomogeneous coordinate of a point in $\mathbf{P}^{1}$. Therefore, we can associate with $z$ the map $\phi$ defined by $s \rightarrow(1, z(s))=\mathbf{z}(s)$. In this case we have $\left[\mathbf{z}, \mathbf{z}^{\prime}\right]=z^{\prime},\left[\mathbf{z}, \mathbf{z}^{\prime \prime}\right]=z^{\prime \prime},\left[\mathbf{z}, \mathbf{z}^{\prime \prime \prime}\right]=z^{\prime \prime \prime},\left[\mathbf{z}^{\prime}, \mathbf{z}^{\prime \prime}\right]=0$, and the curvature becomes [24]

$$
k=\frac{1}{2}\{z, s\}
$$

For an arbitrary physical state with potential function $W$ we have

$$
W=-\frac{\hbar^{2}}{4 m}\left\{e^{(2 i / \hbar) S_{0}}, q\right\}=-\frac{\hbar^{2}}{2 m} k_{W},
$$

and similarly for the quantum potential

$$
Q=\frac{\hbar^{2}}{4 m}\left\{S_{0}, q\right\}=\frac{\hbar^{2}}{2 m} k_{\mathrm{Q}}
$$

where $k_{W}$ is the curvature associated with the map

$$
q \longrightarrow\left(1, e^{(2 i / \hbar) S_{0}(q)}\right)
$$

while the curvature $k_{\mathrm{Q}}$ is associated with the map

$$
q \longrightarrow\left(1, S_{0}(q)\right)
$$

The function defining the map (72) coincides with the trivialising map, whereas the Schwarzian identity (12) can be now seen as difference of curvatures

$$
\left(\partial_{q} S_{0}\right)^{2}=\hbar^{2} k_{W}-\hbar^{2} k_{Q}
$$

The QSHJE (7) can be written in the form

$$
\frac{1}{2 m}\left(\frac{\partial S_{0}(q)}{\partial q}\right)^{2}+W(q)+\frac{\hbar^{2}}{2 m} k_{\mathrm{Q}}=0 .
$$

We therefore note that in the one-dimensional QSHJE the quantum potential is interpreted as a curvature and is an intrinsically quantum characteristic of the particle. In higher dimension the curvature term takes the form

$$
Q=-\frac{\hbar^{2}}{2 m} \frac{\Delta R}{R}
$$


and the corresponding form in the relativistic case. The continuity condition, (32), implies that

$$
R^{2} \partial_{i} S_{0}=\epsilon_{i}^{i_{2} \cdots i_{D}} \partial_{i_{2}} F_{i_{3} \cdots i_{D}}
$$

where $F$ is a $(D-2)$ form. The $3 D$ case $R^{2} \partial_{i} S_{0}$ is the curl of a vector, denoted by $B$,

$$
R^{2} \nabla S_{0}=\nabla \times B
$$

Hence, the QSHJE, (7), takes the form

$$
(\nabla \times B)^{2}=\hbar^{2} R^{3} \Delta R+2 m E R^{4} .
$$

In the time-dependent relativistic case $F$ is a $(D-1)$-form. We have

$$
R^{2}\left(\partial_{\mu} S-e A_{\mu}\right)=\epsilon_{\mu}^{\sigma_{1} \cdots \sigma_{D}} \partial_{\sigma_{1}} F_{\sigma_{2} \cdots \sigma_{D}}=\partial^{\nu} B_{\mu \nu}
$$

that is

$$
R^{2}=\frac{\left(\partial^{\mu} S-e A^{\mu}\right)}{(\partial S-e A)^{2}} \partial^{\nu} B_{\mu \nu} .
$$

In terms of $B$ and $R$ the RQHJE, which correspond to the real part of (46) with $\alpha=i / \hbar$, takes the form

$$
\partial^{\nu} B_{\mu \nu} \partial_{\sigma} B^{\mu \sigma}+R^{4} m^{2} c^{2}-\hbar^{2} R^{3} \square R=0 .
$$

Thus, in the higher dimensional case the quantum potential corresponds to the curvature of the function $R(q)$. We can investigate the properties of the curvature term by studying the free one-dimensional particle, with $W^{0}\left(q^{0}\right) \equiv 0$. In this case the Schrödinger equation takes the form

$$
\frac{\partial^{2}}{\partial q^{2}} \psi=0
$$

with the two linearly independent solutions being $\psi^{D}=q^{0}$ and $\psi=$ const. As discussed previously an essential tenant of the equivalence postulate formalism is that both solutions of the Schrödinger equation must be retained. The linear combination of $\psi$ and $\psi^{D}, \psi^{D}+i \ell \psi$, appears in the solution for $S_{0}$, (16). In the case of the state $W^{0}\left(q^{0}\right) \equiv 0$, with $\psi^{D}=q^{0}$ and $\psi=1$, this combination reads $q^{0}+i \ell_{0}$, and $\ell_{0} \equiv \ell$ should have the dimension of length. On the other hand, from the fact that by (22) the trivialising coordinate $q^{0}$ is given by the ratio $\psi^{D} / \psi$ up to a Möbius transformation, it follows that $\ell$ and $w=\psi^{D} / \psi$ have the dimension of length for any state. The reduced action $S_{0}^{0}$ corresponding to the trivial state $W^{0}$ is

$$
\mathrm{e}^{(2 i / \hbar) S_{0}^{0}}=\mathrm{e}^{i \alpha} \frac{q^{0}+i \bar{\ell}_{0}}{q^{0}-i \ell_{0}},
$$

and the conjugate momentum $p_{0}=\partial_{q^{0}} S_{0}^{0}$ has the form

$$
p_{0}= \pm \frac{\hbar\left(\ell_{0}+\bar{\ell}_{0}\right)}{2\left|q^{0}-i \ell_{0}\right|^{2}} \text {. }
$$

It follows that $p_{0}$ vanishes only for $q^{0} \rightarrow \pm \infty$ and is maximised at $q^{0}=-\operatorname{Im} \ell_{0}$

$$
\left|p_{0}\left(-\operatorname{Im} \ell_{0}\right)\right|=\frac{\hbar}{\operatorname{Re} \ell_{0}} .
$$

Since $\operatorname{Re} \ell_{0} \neq 0, p_{0}$ is always finite, and $\ell_{0}$ provides an ultraviolet cutoff. This property extends to any state [8-14]. It follows that the equivalence postulate implies an ultraviolet cutoff on the conjugate momentum. We can contemplate the potential form of this ultraviolet cutoff by studying the classical limit,

$$
\lim _{\hbar \rightarrow 0} p_{0}=0
$$

From (85) it follows that $\operatorname{Im} \ell_{0}$ can be absorbed by a shift of $q^{0}$. Hence, in the limit equation (87) we can set $\operatorname{Im} \ell_{0}=0$ and distinguish the cases $q^{0}=0$ and $q^{0} \neq 0$. Let us define $\gamma$ by

$$
\operatorname{Re} \ell_{0} \underset{\hbar \rightarrow 0}{ } \hbar^{\gamma} \text {. }
$$

Then

$$
p_{0} \widetilde{\hbar \rightarrow 0} \begin{cases}\hbar^{\gamma+1}, & q_{0} \neq 0, \\ \hbar^{1-\gamma}, & q_{0}=0,\end{cases}
$$

and by (87)

$$
-1<\gamma<1
$$

A constant length having powers of $\hbar$ can be constructed by means of the Compton length, $\lambda_{c}=\hbar /(m c)$, and the Planck length, $\lambda_{p}=\sqrt{\hbar G / c^{3}}$. It is also noted that a constant length which is independent of $\hbar$ is provided by $\lambda_{e}=e^{2} / m c^{2}$ where $e$ is the electric charge. Thus $\ell_{0}$ can be considered as a suitable function of $\lambda_{c}, \lambda_{p}$, and $\lambda_{e}$ satisfying the constraint (90). Of the three constants it is noted that $\lambda_{p}$ satisfies the condition (90), whereas $\lambda_{c}$ and $\lambda_{e}$ do not. Therefore, we can set

$$
\operatorname{Re} \ell_{0}=\lambda_{p}=\sqrt{\frac{\hbar G}{c^{3}}}
$$

With this choice of $\operatorname{Re} \ell_{0}$, by (86), the maximum of $\left|p_{0}\right|$ is given by

$$
\left|p_{0}\left(-\operatorname{Im} \ell_{0}\right)\right|=\sqrt{\frac{\hbar c^{3}}{G}} .
$$

The quantum potential associated with the state $W^{0}$ is given by

$$
Q^{0}=\frac{\hbar^{2}}{4 m}\left\{S_{0}^{0}, q^{0}\right\}=-\frac{\hbar^{2}\left(\operatorname{Re} \ell_{0}\right)^{2}}{2 m} \frac{1}{\left|q^{0}-i \ell_{0}\right|^{4}}
$$

We can make naive estimates of this potential. Taking $m \sim$ $100 \mathrm{GeV} ; \operatorname{Re} \ell_{0}=\lambda_{p} \approx 10^{-35} \mathrm{~m}$; and $q^{0}$ as the size of the observable universe $q^{0} \sim 93$ Ly gives $|Q| \sim 10^{-202} \mathrm{eV}$. A tiny amount of energy indeed! It is noted that the sign of $Q$ is 
negative in the one-dimensional case. However, there is no reason to expect that this will be the case in higher dimensions as the curvature of the function $R(q)$ is, in principle, undetermined. It should be emphasised that this is a naive analysis, reflecting the appearance of a length scale in the formalism associated with the intrinsic properties of particle and providing an internal energy component similar to its mass. In this estimate I have taken the particle mass to be $100 \mathrm{GeV}$ and $q$ to be given by the size of the observable universe, and the quantum potential is that corresponding to the state $W^{0}$. Taking $q \sim 1 \mathrm{~m}$ yields $|Q| \sim 10^{-96} \mathrm{eV}$. In this respect a relevant question is what is the effective $q$ that one should take. A consistency condition of the equivalence postulate formalism dictates that the trivialising transformation is continuous on the extended real line [8-14], that is, at $q= \pm \infty$. The question therefore is what is the effective $q= \pm \infty$ from the elementary particle perspective. A more detailed analysis can take into account relativistic particles by considering the relativistic quantum Hamilton-Jacobi equation and by considering particles with constant, but finite, $W$ functions. It is noted that several authors considered the possibility of interpreting the quantum potential in Bohmian mechanics as the source of dark energy $[25,26]$, as well as detailed cosmological scenarios [27].

\section{The Multiparticle Case}

So far the single particle case was discussed. It is interesting to study the question of the multiparticle case and whether the quantum potential is additive in this case. Furthermore, while the single particle case can reveal basic properties of the mathematical formalism, the physical case involves the multi-particle state, as it requires at least an observer and an observee. In the case of two free particles of energy $E$ and masses $m_{1}$ and $m_{2}$, the QSHJE reads

$$
\begin{aligned}
\frac{1}{2 m_{1}} & \left(\nabla_{1} S_{0}\right)^{2}+\frac{1}{2 m_{2}}\left(\nabla_{2} S_{0}\right)^{2}-E \\
& -\frac{\hbar^{2}}{2 m_{1}} \frac{\Delta_{1} R}{R}-\frac{\hbar^{2}}{2 m_{2}} \frac{\Delta_{2} R}{R}=0 .
\end{aligned}
$$

The continuity equation is

$$
\frac{1}{m_{1}} \nabla_{1} \cdot\left(R^{2} \nabla_{1} S_{0}\right)+\frac{1}{m_{2}} \nabla_{2} \cdot\left(R^{2} \nabla_{2} S_{0}\right)=0
$$

Next, we set

$$
r=r_{1}-r_{2}, \quad r_{\mathrm{c} . \mathrm{m} .}=\frac{m_{1} r_{1}+m_{2} r_{2}}{m_{1}+m_{2}}, \quad m=\frac{m_{1} m_{2}}{m_{1}+m_{2}},
$$

where $r_{1}$ and $r_{2}$ are the vectors of the two particles. With respect to the new variables (94) and (95) have the form

$$
\begin{gathered}
\frac{1}{2\left(m_{1}+m_{2}\right)}\left(\nabla_{r_{\text {c. } \mathrm{m} .}} S_{0}\right)^{2}+\frac{1}{2 m}\left(\nabla S_{0}\right)^{2}-E \\
-\frac{\hbar^{2}}{2\left(m_{1}+m_{2}\right)} \frac{\Delta_{r_{\mathrm{c} . \mathrm{m} .}} R}{R}-\frac{\hbar^{2}}{2 m} \frac{\Delta R}{R}=0, \\
\frac{1}{m_{1}+m_{2}} \nabla_{r_{\mathrm{c} . \mathrm{m} .}} \cdot\left(R^{2} \nabla_{r_{\mathrm{c} . \mathrm{m} .}} S_{0}\right)+\frac{1}{m} \nabla \cdot\left(R^{2} \nabla S_{0}\right)=0,
\end{gathered}
$$

where $\nabla\left(\nabla_{r_{\text {c.m. }}}\right)$ and $\Delta\left(\Delta_{r_{\text {c.m. }}}\right)$ are the gradient and Laplacian with respect to the components of the vector $r\left(r_{\text {c.m. }}\right)$. These equations can be decomposed into the equations for the centre of mass $r_{\text {c.m. }}$ and those for the relative motion. Reference [28] focussed on the equations for the relative motion and argued that the quantum potential is at the origin of the gravitational interactions. Estimating the energy due to gravitational interaction between two protons at a distance of $1 \mathrm{fm}=10^{-15} \mathrm{~m}$ gives $|E| \sim 10^{-31} \mathrm{eV}$, whereas estimating it from the quantum potential yields $|E| \sim 10^{-33} \mathrm{eV}$, which is not too far off. While this is an intriguing proposition, it seems that the constructive approach to incorporate gravity into the formalism is to extend the cocycle and quadratic identities to curved backgrounds, which is left for future work. Instead I focus here on the equations for motion of the centre of mass, which take the form

$$
\begin{gathered}
\frac{1}{2\left(m_{1}+m_{2}\right)}\left(\nabla_{r_{\text {c. } \mathrm{m} .}} S_{0}\right)^{2}-\widetilde{E}-\frac{\hbar^{2}}{2\left(m_{1}+m_{2}\right)} \frac{\Delta_{r_{\text {c. } \mathrm{m} .}} R}{R}=0, \\
\frac{1}{\left(m_{1}+m_{2}\right)} \nabla_{r_{\text {c.m. }}} \cdot\left(R^{2} \nabla_{r_{\text {c. } \mathrm{m} .}} S_{0}\right)=0 .
\end{gathered}
$$

The main point to note is that the effective mass in the relative motion is the reduced mass, whereas the effective mass in the centre of mass motion is additive, which is what we expect physically. Hence, for large number of particles the mass and potential energies are summed. In the equivalence postulate framework the mass and quantum energy of elementary particles are correlated and are different attributes of the same particle. Finally, the same arguments could have been presented by using the relativistic form of the equations. However, nonrelativistic treatment is viable given that the majority of matter in the universe is expected to be nonrelativistic.

\section{Conclusions}

Contemporary observations at the smallest and highest length scales are well accounted for by the standard model of particle physics and by general relativity, respectively. Yet the synthesis of these two basic theories remains elusive. By hypothesising that all physical systems labelled by a potential function $W(q)$ are connected by coordinate transformations, the equivalence postulate of quantum mechanics offers an axiomatic starting point for formulating quantum field theories and quantum gravity. A somewhat heuristic view of the equivalence postulate procedure is the following. In the classical Hamilton-Jacobi formalism one seeks a transformation from an a nontrivial Hamiltonian to a trivial Hamiltonian. The transformations are canonical and treat the phase space variables as independent variables. This ensures that the classical equations of motion are preserved. It ensures that the classical path is selected. The key property of quantum mechanics is that the phase space variables are not independent but satisfy the basic quantum mechanical relation

$$
[\widehat{q}, \widehat{p}]=i \hbar .
$$


Therefore, we can view the equivalence postulate procedure as implementing the Hamilton-Jacobi trivialisation algorithm, but taking the phase space variables to be related by the generating function $S(q)$, via relation (4). The solution to this problem is not given by the CSHJE, (3), but rather by the QSHJE, (7). Covariance of the QSHJE then implies that all physical systems labelled by the potential function $W(q)$ are connected by the coordinate transformations. This view is in line with the picture that quantum mechanically all possible paths are allowed, and we may imagine that different paths correspond to different $W(q)$ labels. The equivalence postulate formalism is encoded in two basic identities. The first is the cocycle condition, (9), which embodies the symmetry properties underlying quantum mechanics in this formalism. The second is the quadratic identity, (12), which is the manifestation of the quantum Hamilton Jacobi equation and is compatible with the equivalence hypothesis. Both of these key ingredients admit suitable generalisations in higher dimensions with Euclidean or Minkowski metrics. A key role in this approach is played by the various dualities [8-14, 29], which are implemented as Legendre transformations. These Legendre dualities represent a complementary facet of the underlying physical principles. In this respect they show that the different physics variables used to characterise the physical systems are related by the dualities and none is superior to the other.

In view of the observational supremacy of the standard model and general relativity in their respective domains, we may question how the equivalence postulate formalism is manifested observationally. Given that quantum gravity effects are likely to be notoriously small, we may expect them to generate deviations in the most extreme regimes of contemporary observations. Indeed, recently the OPERA collaboration announced intriguing results on the superluminal propagation of neutrinos [30]. While the fate of the results of this experiment is in doubt [31], it is clear that the neutrino sector will provide experimental data that will continue to probe the validity of the standard model. In the context of the equivalence postulate formalism it was shown in [32-36] that the quantum potential in this formalism leads to dispersion relations that modify the classical relativistic energymomentum relation. Such modifications of the relativistic energy-momentum relation are expected in different approaches to quantum gravity and the relevant question is whether they are sufficiently large to be observable. In this paper I proposed that the quantum potential that arises in the equivalence postulate formulation can be regarded as an intrinsic curvature term, which characterises elementary particles, and may be interpreted as dark energy.

\section{Acknowledgments}

The author would like to thank the theoretical Physics Department at Oxford University for hospitality. This work was supported in part by the STFC (PP/D000416/1).

\section{References}

[1] A. E. Faraggi, D. V. Nanopoulos, and K. Yuan, "A standard-like model in the four-dimensional free fermionic string formulation," Nuclear Physics B, vol. 335, no. 2, pp. 347-362, 1990.

[2] A. E. Faraggi, "A new standard-like model in the four dimensional free fermionic string formulation," Physics Letters B, vol. 278, no. 1-2, pp. 131-139, 1992.

[3] A. E. Faraggi, "Construction of realistic standard-like models in the free fermionic superstring formulation," Nuclear Physics B, vol. 387, no. 2, pp. 239-262, 1992.

[4] G. B. Cleaver, A. E. Faraggi, and D. V. Nanopoulos, "String derived MSSM and M-theory unification," Physics Letters B, vol. 455, no. 1-4, pp. 135-146, 1999.

[5] K. Christodoulides, A. E. Faraggi, and J. Rizos, “Top quark mass in exophobic Pati-Salam heterotic string model," Physics Letters $B$, vol. 702, no. 1, pp. 81-89, 2011.

[6] A. Giveon, M. Porrati, and E. Rabinovici, "Target space duality in string theory," Physics Reports, vol. 244, no. 2-3, pp. 77-202, 1994.

[7] C. M. Hull, "A geometry for non-geometric string backgrounds," Journal of High Energy Physics, vol. 2005, no. 10, article 065, 2005.

[8] A. E. Faraggi and M. Matone, "Quantum mechanics from an equivalence principle," Physics Letters B, vol. 450, no. 1-3, pp. 34-40, 1999.

[9] A. E. Faraggi and M. Matone, "The equivalence principle of quantum mechanics: uniqueness theorem," Physics Letters B, vol. 437, no. 3-4, pp. 369-380, 1998.

[10] A. E. Faraggi and M. Matone, "Quantum transformations," Physics Letters A, vol. 249, no. 3, pp. 180-190, 1998.

[11] A. E. Faraggi and M. Matone, "Equivalence principle, Planck length and quantum Hamilton-Jacobi equation," Physics Letters $B$, vol. 445, no. 1-2, pp. 77-81, 1998.

[12] A. E. Faraggi and M. Matone, "Equivalence principle: tunnelling, quantized spectra and trajectories from the quantum HJ equation," Physics Letters B, vol. 445, no. 3-4, pp. 357-365, 1999.

[13] A. E. Faraggi and M. Matone, "The equivalence postulate of quantum mechanics," International Journal of Modern Physics $A$, vol. 15, no. 13, p. 1869, 2000.

[14] A. E. Faraggi and M. Matone, "The equivalence postulate of quantum mechanics: main theorems," in Quantum Trajectories, P. Chattaraj, Ed., pp. 17-39, Taylor\&Francis/CRC Press, 2011.

[15] G. Bertoldi, A. E. Faraggi, and M. Matone, "Equivalence principle, higher-dimensional Möbius group and the hidden antisymmetric tensor of quantum mechanics," Classical and Quantum Gravity, vol. 17, no. 19, pp. 3965-4005, 2000.

[16] E. R. Floyd, "Bohr-Sommerfeld quantization with the effective action variable," Physical Review D, vol. 25, no. 6, pp. 1547-1551, 1982.

[17] E. R. Floyd, "Modified potential and Bohm's quantummechanical potential," Physical Review D, vol. 26, no. 6, pp. 1339-1347, 1982.

[18] E. R. Floyd, "Arbitrary initial conditions of nonlocal hidden variables," Physical Review D, vol. 29, no. 8, pp. 1842-1844, 1984.

[19] E. R. Floyd, "Closed-form solutions for the modified potential," Physical Review D, vol. 34, no. 10, pp. 3246-3249, 1986.

[20] E. R. Floyd, "The Ermakov invariant for the trajectory representation of quantum mechanics," Physics Letters A, vol. 214, no. 5-6, pp. 259-265, 1996. 
[21] E. R. Floyd, "Classical limit of the trajectory representation of quantum mechanics, loss of information and residual indeterminacy," International Journal of Modern Physics A, vol. 15, no. 9, p. 1363, 2000.

[22] R. E. Wyatt, Quantum Dynamics with Trajectories: Introduction to Quantum Hydrodynamics, vol. 28 of Interdisciplinary Applied Mathematics, Springer, New York, NY, USA, 2005.

[23] B. Poirier, "Reconciling semiclassical and Bohmian mechanics. I. Stationary states," Journal of Chemical Physics, vol. 121, no. 10, p. $4501,2004$.

[24] H. Flanders, "The Schwarzian as a curvature," Journal of Differential Geometry, vol. 4, pp. 515-519, 1970.

[25] P. F. Gonzalez-Diaz, "Axion phantom energy," Physical Review D, vol. 69, no. 6, Article ID 063522, 6 pages, 2004.

[26] P. F. Gonzalez-Diaz and A. Rozas-Fernandez, "Accelerating Hilbert-Einstein universe without dynamic dark energy," Physics Letters B, vol. 641, no. 2, pp. 134-138, 2006.

[27] J. Marto and P. Vargas-Moniz, "de Broglie-Bohm FRW universes in quantum string cosmology," Physical Review D, vol. 65, no. 2, Article ID 023516, 16 pages, 2001.

[28] M. Matone, "Equivalence postulate and quantum origin of gravitation," Foundations of Physics Letters, vol. 15, no. 4, pp. 311328, 2002.

[29] A. E. Faraggi and M. Matone, "Duality of $x$ and $\psi$ and a statistical interpretation of space in quantum mechanics," Physical Review Letters, vol. 78, no. 2, pp. 163-166, 1997.

[30] T. Adam, N. Agafonova, A. Aleksandrov et al., "Measurement of the neutrino velocity with the OPERA detector in the CNGS beam," Journal of High Energy Physics, vol. 2012, no. 10, article 93, 2012.

[31] M. Antonello, P. Aprili, B. Baibussinov et al., "Measurement of the neutrino velocity with the ICARUS detector at the CNGS beam," Physics Letters B, vol. 713, no. 1, pp. 17-22, 2012.

[32] M. Matone, "Superluminality and a curious phenomenon in the relativistic quantum Hamilton-Jacobi equation," Physica Scripta, vol. 86, no. 5, Article ID 055007, 2012.

[33] M. Matone, ““Thermodynamique cachée des particules” and the quantum potential," Annales Fond Louis de Broglie, vol. 37, pp. 177-185, 2012.

[34] A. E. Faraggi, "Superluminality and the equivalence postulate of quantum mechanics," The European Physical Journal C, vol. 72, p. 1944, 2012.

[35] A. E. Faraggi, "OPERA data and the equivalence postulate of quantum mechanics," The European Physical Journal C, vol. 72, p. 1944, 2012.

[36] E. R. Floyd, "OPERA superluminal neutrinos per quantum trajectories," http://arxiv.org/abs/1112.4779 . 

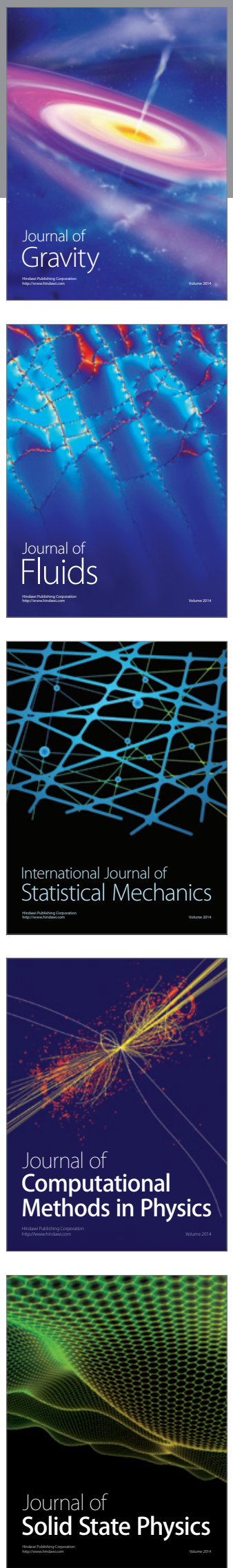

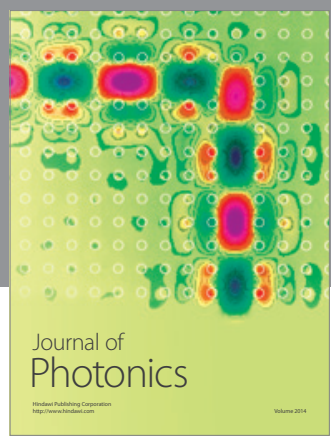

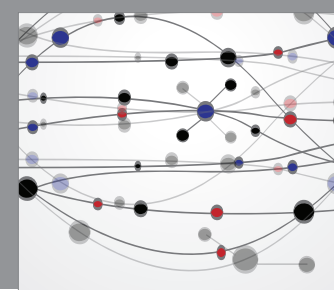

The Scientific World Journal

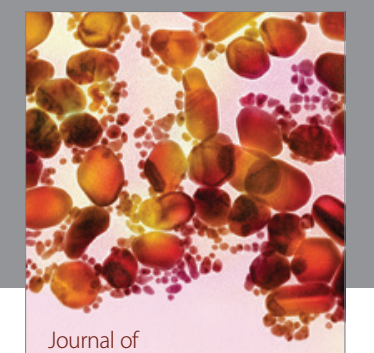

Soft Matter
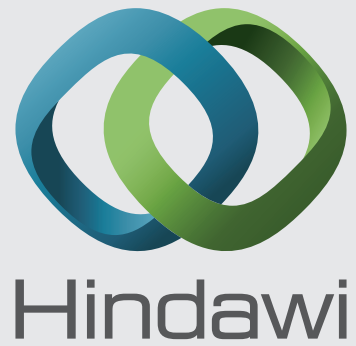

Submit your manuscripts at

http://www.hindawi.com
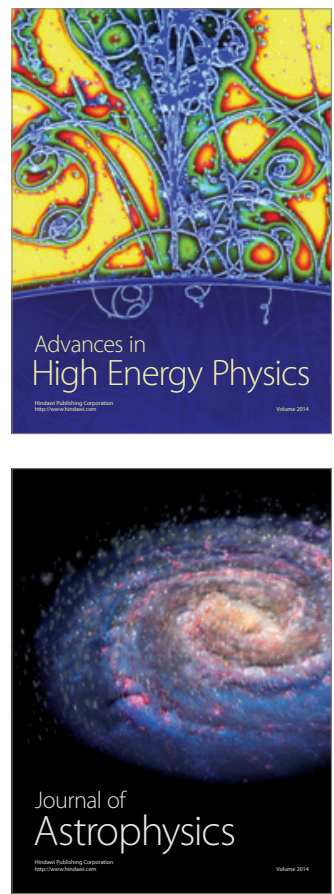
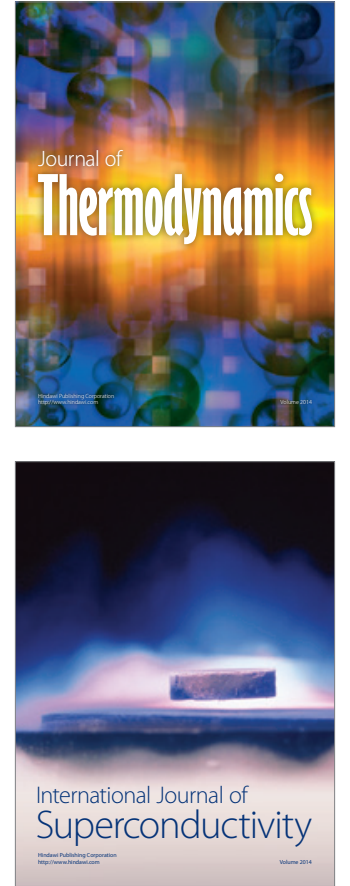
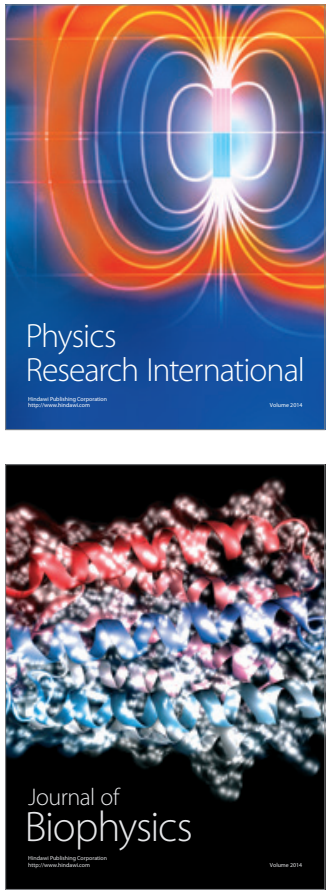
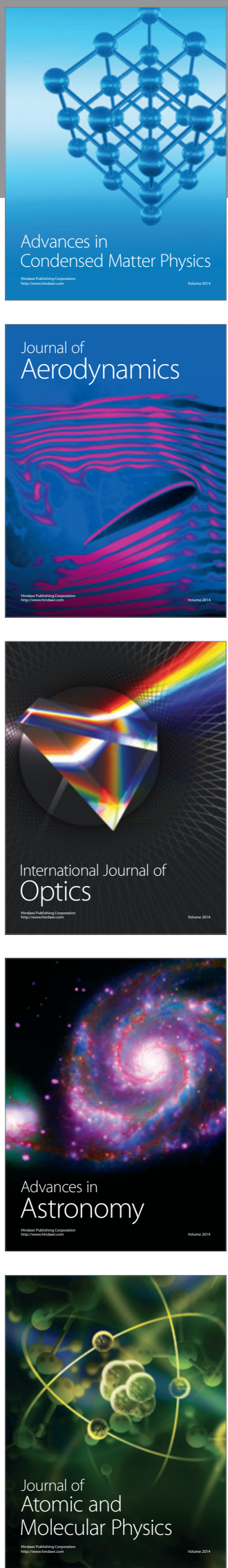